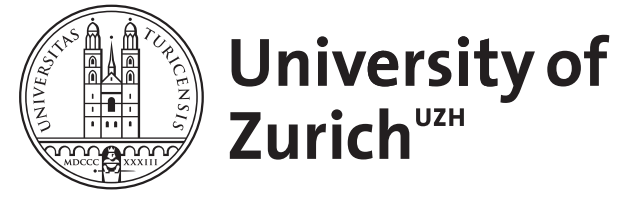
Archive

University of Zurich

University Library

Strickhofstrasse 39

CH-8057 Zurich

www.zora.uzh.ch

Year: 2014

\title{
Neuro-ophthalmology update
}

Weber, Konrad P ; Straumann, Dominik

DOI: https://doi.org/10.1007/s00415-013-7105-x

Posted at the Zurich Open Repository and Archive, University of Zurich ZORA URL: https://doi.org/10.5167/uzh-81444

Journal Article

Accepted Version

Originally published at:

Weber, Konrad P; Straumann, Dominik (2014). Neuro-ophthalmology update. Journal of Neurology, 261(7):1251-1256.

DOI: https://doi.org/10.1007/s00415-013-7105-x 


\title{
Neuro-Ophthalmology Update
}

\author{
Konrad P. Weber, $\mathrm{MD}^{1,2}$; Dominik Straumann ${ }^{2}$, MD. \\ ${ }^{1}$ Department of Ophthalmology, University Hospital Zurich, Zurich, Switzerland. \\ ${ }^{2}$ Department of Neurology, University Hospital Zurich, Zurich, Switzerland.
}

Word count: 3260

References: 29

1 table, 1 highlights box

Corresponding author:

Konrad P. Weber, MD

University Hospital Zurich

Department of Ophthalmology

Frauenklinikstrasse 24

CH-8091 Zürich

Switzerland

Phone: +414425511 11

Fax: $\quad+41442554349$

Mail: konrad.weber@usz.ch

\section{Disclosure}

The authors declare no conflict of interest.

\begin{abstract}
This review summarizes the most relevant articles from the field of neuro-ophthalmology published in the Journal of Neurology from January 2012 to July 2013. With the advent of video-oculography, several articles describe new applications for eye movement recordings as a diagnostic tool for a wide range of disorders. In myasthenia gravis, anti-Kv1.4 and anti-Lrp4 have been characterized as promising novel autoantibodies for the diagnosis of hitherto 'seronegative' myasthenia gravis. Several articles address new diagnostic and therapeutic approaches to neuromyelitis optica (NMO), which further sharpen its profile as a distinct entity. 4-aminopyridine has become a standard therapeutic for patients with cerebellar downbeat nystagmus. Finally, revised diagnostic criteria have been proposed for chronic relapsing inflammatory optic neuropathy (CRION) based on a careful literature review over the last decade.
\end{abstract}

\section{Keywords}

Neuro-ophthalmology, eye movements, ocular myasthenia gravis, optic neuritis, neuromyelitis optica (NMO), chronic relapsing inflammatory optic neuropathy (CRION), cerebellar downbeat nystagmus, 4-aminopyridine.

\section{Introduction}

The eclectic field of neuro-ophthalmology keeps evolving rapidly. In this article we are summarizing the most relevant publications in the field published in the Journal of Neurology from January 2012 to July 2013. The review is divided into sections about the use of eye movement measurements as a probe of disease, novel autoantibodies for the diagnosis of myasthenia gravis, neuromyelitis optica (NMO) and chronic relapsing inflammatory optic neuropathy (CRION) and the therapeutic use of 4-aminopyridine. 


\section{Eye movements as a probe of disease}

The examination of eye movements is a rich source of clinical information to neuroophthalmologists [1]. Thanks to detailed knowledge of the underlying neurophysiological circuits in the brainstem, eye movements provide precise information about the localization of disease and often valuable clues about its etiology. With the advent of video-oculography, the quantitative analysis of eye movements now becomes readily accessible to clinics.

Tseng et al. devised a universally applicable patient screening tool based on automated analysis of natural viewing eye movements [2]. The participants simply had to watch television while their eye movements were recorded with a head-mounted video-based eye tracker. The eye movements were then analyzed for ocular motor features such as saccade metrics, as well as visual attention based on saliency maps. The investigators analyzed patient populations from two different age groups: elderly people with Parkinson's disease as well as children with attention deficit hyperactivity disorder (ADHD) and fetal alcohol spectrum disorder (FASD). Based on eye movement recordings from only $15 \mathrm{~min}$ of video, the method was able to distinguish patients with Parkinson's from age-matched controls with an accuracy of $89.6 \%$ and ADHD versus FASD children with $77.3 \%$ accuracy. While in Parkinson's the distinction was made based on ocular motor features, ADHD and FASD children were distinguished based on visual attention features. The patient-friendly nature of this automated test makes it a promising screening tool for large populations.

In a population-based incidence cohort, Linder et al. looked at eye movements in patients with newly diagnosed Parkinson syndrome before treatment [3]. They examined 105 patients with Parkinson's disease, 11 patients with progressive supranuclear palsy (PSP) and 19 patients with multiple system atrophy with predominant Parkinsonism (MSA-P). The diagnosis was established by a movement disorder specialist and supported by single-photon emission computed tomography (FP-CIT SPECT). Analysis of square-wave jerks, horizontal and vertical saccades and smooth pursuit revealed pathological ocular motor function in all patient groups. The ocular motor testing, however, could not reliably differentiate different subtypes of Parkinson syndrome early in the course of the disease. Nevertheless, some features, such as slow vertical saccade velocity or low smooth pursuit gain, were found to be indicative of PSP.

Ong and colleagues developed Read-Right, a free web-based application (see www.readright.ucl.ac.uk) to improve reading in patients with right-sided hemianopia [4]. First, the web application automatically assessed the visual field of the patients. Then reading speed was measured with six standardized paragraphs of static text at baseline and after every 5 hours of training. Reading training consisted of freely chosen leftward scrolling text that could be adjusted in speed and color. The rationale of the therapy was to induce involuntary saccades into the patient's blind right hemifield as a strategy to improve reading when returning to normal, static text. With this paradigm, the authors could demonstrate a significant, training-dependent improvement of reading speeds of up to $46 \%$ after 20 hours of training. The free website provides a promising approach to deliver reading training to the home of patients when rehabilitation resources are scarce.

Predicting consciousness recovery in acute patients with vegetative state (VS) is a diagnostic challenge on intensive care units (ICU). Weiss et al. investigated the prognostic value of caloric bedside irrigation in 26 brain-injured patients with VS [5]. They found that the presence of quick phases (rather than slow phases) of caloric nystagmus has a very high positive predictive value of 0.93 for regaining consciousness in these patients. The authors explain the good correlation with the close anatomical relation between the pathways of the 
vestibulo-ocular reflex and consciousness in the brainstem. This indicator of a favorable outcome may be very helpful for guiding therapeutic decisions on ICU. In another study, Trojano et al. explored the visual tracking behavior of chronic patients with disorders of consciousness [6]. Their goal was to distinguish patients with VS from patients with minimally conscious state, who have a more favorable outcome. The authors found that patients with minimally conscious state fixated significantly more on presented targets, while fixation in patients with VS was at chance level. Quantitative analysis of visual tracking behavior, as proposed by the authors, may therefore be a promising tool for assessing and following up patients with impaired consciousness.

Fatigue is one of the most common symptoms of multiple sclerosis (MS) with a substantial impact on the quality of life of patients. Fatigue is usually assessed with the fatigue severity scale (FSS), but objective measures of fatigue are lacking. Finke et al. set out to quantify fatigue in MS patients with a saccadic fatigue task [7]. They asked MS patients with and without fatigue to continuously perform saccades over a period of 10 minutes. They found that patients with fatigue had a significantly larger decrease of saccade peak velocity and amplitude than patients without fatigue. For their study they used a portable infrared eyemovement recording device, which would allow for convenient bedside testing of fatigue in a clinical setting. The system, however, only provides 'cyclopean' recordings of the average position of both eyes. As the authors acknowledge, the presence of internuclear ophthalmoplegia (INO), which is a frequent finding in MS, may interfere with the result in these patients.

\section{Novel myasthenia gravis autoantibodies}

Ocular myasthenia gravis (MG) is often a diagnostic dilemma, because autoantibodies to acetylcholine receptors (AChR) are negative in over $50 \%$ of cases, repetitive nerve stimulation of peripheral muscles has a poor sensitivity, and single fiber electromyography as the current gold standard is cumbersome and uncomfortable for the patients. For these reasons, detection of additional autoantibodies would be very helpful in bridging the diagnostic gap of hitherto 'seronegative' ocular MG.

Recently, novel autoantibodies to Kv1.4 have been identified [8]. Kv1.4 is a subunit of the voltage-gated potassium channel (VGKC) involved in the presynaptic release of acetylcholine. The study has been done in a Japanese population, where the antibodies were associated with severe MG with bulbar involvement, myasthenic crisis, thymoma, myocarditis, and QT prolongation on electrocardiogram. Based on this study, Romi et al. set out to investigate the clinical features and serological associations of anti-Kv1.4 antibodies in a Caucasian MG population [9]. They included samples from 129 myasthenia gravis patients with mainly ocular symptoms. They could detect anti-Kv1.4 antibodies in $17 \%$ of their patients, most of them women with late-onset MG. So, while in a Japanese population, antiKv1.4 autoantibodies seem to be associated with severe MG and thymoma, in Caucasians the same antibodies are associated with mild, late-onset MG in women. To reconcile this disparity, the authors point out to the genetic differences between the two study populations as a likely explanation.

Approximately $10 \%$ of patients with generalized MG have neither antibodies against AChR nor muscle-specific tyrosine kinase (MuSK). However, onset and progression of these socalled double-seronegative patients is very similar to seropositive patients. As they respond similarly to plasma exchange and immunosuppressive therapy, the disease is likely caused by 
another pathogenic autoantibody. Recently, autoantibodies to low-density lipoprotein receptor-related protein 4 (Lrp4) have been characterized in patients with AChR-negative MG [10]. In this Japanese population, anti-Lrp4 antibodies have been detected in only 9 out of 300 MG patients. Based on this study, Pevzner et al. analyzed sera from 13 double-seronegative MG patients in a Caucasian population [11]. In contrast to the previous study, they detected autoantibodies against Lrp4 much more frequently in about $50 \%$ of their patients (7/13) with a female preponderance and rather late onset. Similar to the differences found for the antiKv1.4 antibodies described above, the authors attributed the variation to the different ethnical background of their patients.

In contrast to double-seronegative MG patients, double-seropositive patients are exceedingly rare with only a handful patients reported to date. Rajakulendran et al. described an interesting case with clinically biphasic MG with both AChR and MuSK antibodies [12]. The patient first presented during adolescence with bulbar predilection typical for AChR-positive MG. Following thymectomy she went into complete remission. 15 years later, she presented again, this time with severe oculobulbar, neck and respiratory muscle involvement. She then had several relapses that had to be treated with intravenous immunoglobulins and plasma exchange. At this stage, the patient was tested positive for both AchR and MuSK antibodies. Although the authors could not prove that the patient was MuSK-negative at initial presentation (as it was not tested), they conjecture from the biphasic clinical course that she switched from a predominantly AchR-mediated to a MuSK-mediated phenotype after thymectomy. Based on their experience, the authors recommend testing for MuSK antibodies in MG patients who relapse after thymectomy as this may substantially change their therapeutical approach.

\section{Neuromyelitis optica (NMO) and chronic relapsing inflammatory optic neuropathy (CRION)}

Neuromyelitis optica (NMO) is a rare inflammatory and demyelinating disease characterized by optic neuritis and longitudinally extensive transverse myelitis. Eugène Devic and his student Fernand Gault coined the term NMO in 1894 based on 16 patients with this rare association of symptoms [13]. However, Jarius et al. re-discovered an earlier, forgotten report from 1804 by the French pathologist and first physician to Louis XVIII, Antoine Portal (1742-1832), who described a patient with visual loss and spinal cord inflammation but no brain pathology [14]. In another paper, Jarius et al. also highlighted the seminal role of Sir Thomas Clifford Allbutt (1836-1925), best known as the inventor of the clinical thermometer, in piecing the NMO syndrome together based on three cases with the same concurrence of symptoms published in 1870 [15].

As NMO is a rare disease, reliable epidemiological data has been quite sparse. Previous studies in different ethnic groups reported variable prevalence rates between 3.2 (Japan) and 44/million (Denmark). Jacob et al. established the epidemiology of NMO in Merseyside, which is a metropolitan county in North West England with the City of Liverpool as its headquarters [16]. The adult population of Merseyside is about 1.1 million with $94.8 \%$ Caucasians, which is approximately representative for the United Kingdom in general. Based on multiple overlapping data sources including hospital records, central anti-aquaporin 4 (AQP4) antibody testing laboratory data and the British Neurological Surveillance Unit, they identified eight cases with NMO or NMO spectrum disorder indicating a prevalence of $7.2 /$ million with an average annual incidence rate of 0.8/million. Extrapolating to the whole UK population, the estimated prevalence would correspond to 365 cases with an average 
annual incidence rate of 39 cases. The authors point out that the relatively low prevalence compared to other studies probably reflects genetic differences in the population studied. Although the authors took great care in avoiding any ascertainment bias by using overlapping data sources, they admit that some NMO cases may have been missed mainly because of misdiagnosis as multiple sclerosis (MS). Due to increasing awareness of NMO and its varied spectrum, the authors expect the prevalence to rise in the future.

To elucidate the time course of NMO, Uzawa et al. retrospectively analyzed a Japanese cohort of 43 patients with a mean disease duration of 14.2 years [17]. Myelitis and optic neuritis were about equally frequent as initial inflammatory events (44.2\% and $41.9 \%)$, while both together occurred in about $9.3 \%$. Interestingly, myelitis was found to be the more frequent initial symptom in older patients ( $>50$ years), whereas optic neuritis was seen more often in younger patients ( $<50$ years). By the end of the observation period, $93 \%$ of the patients had positive anti-AQP4 antibody titers. The median interval from onset until fulfillment of the 2006 NMO criteria [18] was 28 months, indicating the difficulty for early diagnosis of the disease. Based on this diagnostic delay of over 2 years, the authors highlight the need for early diagnostic markers or more specific criteria in order to initiate the appropriate treatment timely.

According to the current diagnostic criteria [18], optic neuritis and myelitis are absolute criteria for NMO. Extension of the myelitis over three or more vertebral segments together with the presence of AQP4 antibodies and a brain MRI that does not meet the criteria for MS further supports the diagnosis of NMO. In a recent study Iorio et al. compared the clinical and neuroimaging characteristics of 37 patients with longitudinally extensive transverse myelitis [19]. 16 of their patients had positive anti-AQP4 antibodies with a higher female to male ratio than the negative patients. In these patients the myelitis was often accompanied by intractable nausea, vomiting and paroxysmal tonic spasms. This was explained by the fact that the inflammatory lesions extended into the brainstem more frequently involving the central grey matter. These distinctive clinical features may help to identify patients with NMO earlier in order to prompt the appropriate treatment.

Despite many similarities with MS, the treatment of NMO is different. Since randomized controlled trials for the treatment of this rare disease are lacking, current therapeutic concepts are still based on expert opinion and consensus criteria [20]. While current best practice recommends oral treatment with azathioprine in combination with corticosteroids, the efficacy of cyclosporine A has not yet been evaluated in NMO. Kageyama et al. reviewed the clinical records of 52 patients with NMO spectrum disorders and included 22 patients for analysis of their treatment [21]. First, the authors confirmed the efficacy of azathioprine in combination with corticosteroids as a treatment for NMO. Second, they provided the first evidence that the combination of cyclosporine A and corticosteroids may be equally effective. Under treatment with cyclosporine A, they observed a significant reduction in the annual relapse rate as well as a decreased dose of concomitantly administered corticosteroids. In summary, the case series provides the first retrospective evidence for the efficacy of cyclosporine $\mathrm{A}$ in the treatment of NMO spectrum disorders, but randomized controlled treatment trials are warranted to confirm these results.

In a case report, Juryńczyket al. reported the deleterious effect of natalizumab in a patient with initially unrecognized NMO [22]. The woman was diagnosed with MS at the age of 22. She had recurrent relapses of spastic paraparesis, sensory deficits and severe right optic neuritis. Over the years, she was treated with interferon B-1a, glatiramer acetate and mitoxantrone. While she was in a stable condition, she was then started on natalizumab. Six 
days after the fourth infusion, she suddenly developed a severe relapse with confusion, vomiting and paralysis of her left upper limb. Her brain MRI showed an extensive, noncontrast-enhancing T2-hyperintense lesion. Negative JC virus PCR ruled out progressive multifocal leucencephalopathy. Based on the presence of anti-AQP4 antibodies, the patient was finally diagnosed with NMO according to the current criteria. Treatment with plasmapheresis and intravenous methylprednisolone led to clinical improvement with resolution of confusion after two weeks. In line with previous reports, this clinical case highlights that, following a few infusions, natalizumab may induce a severe relapse in patients with NMO. The authors therefore recommend testing for anti-AQP4 antibodies in atypical MS patients, especially with severe spinal and/or optic nerve involvement, before natalizumab initiation and in patients who develop extensive brain lesions during treatment.

Horwitz et al investigated the diagnostic value of a lumbar puncture in patients with optic neuritis depending on the result of visual evoked potential (VEP) and MRI examination of the brain [23]. For this purpose, they retrospectively analyzed 437 patients who presented with optic neuritis without known MS prior to referral. They found that cerebrospinal fluid (CSF) abnormalities (elevated leucocytes, elevated IgG index or oligoclonal bands) correlated strongly with pathological VEP and MRI results $(\mathrm{p}<0.0001)$. Vice versa, they calculated that patients with both normal MRI and VEP had a $96 \%$ probability of normal CSF findings. From their results they concluded that the diagnostic contribution of a lumbar puncture to the evaluation of optic neuritis is negligible in patients with normal VEP and MRI. Therefore, the authors suggested that all patients with suspected optic neuritis should be evaluated with VEP and MRI before deciding on a lumbar puncture.

Apart from NMO, the differential diagnosis of optic neuritis includes the rare entity of chronic relapsing inflammatory optic neuropathy (CRION), which was first described by Kidd et al. in 2003 [24]. In a systematic literature review, Petzold et al. now analyzed 122 patients with CRION over the last decade [25]. They characterized it as a rare, worldwide disease with a peak in the fourth decade and a female preponderance. Typically, patients presented with bilateral simultaneous or sequential, mostly severe visual loss and at least one relapse. Pain at onset was reported in at least about a third of the cases and particularly recurrence of pain after reducing or stopping the steroids was noted as a red flag. Importantly, most of the patients were negative for AQP4 antibodies, suggesting that CRION is an entity distinct from NMO. In addition, MRI scans of the brain did not show demyelinating lesions typical for MS. Taken together, its etiology still remains unknown, but seems likely to be immune-mediated. All patients treated with methylprednisolone responded, but relapsed after stopping the treatment. Therefore, oral steroids had to be carefully tapered under rigorous follow-up to identify the individual minimal effective dose. As a consequence of the steroiddependency, steroid-sparing agents (azathioprine, methotrexate, cyclophosphamide or mycophenolate) had to be used in many cases. But as a silver lining, in the experience of the authors, disease activity sometimes settled over a decade. Based on their careful review with the key points outlined above, they proposed revised diagnostic criteria for CRION (table 1). Since the diagnosis of CRION based on these criteria remains mainly clinical without any specific blood biomarker, the authors hope for the discovery of a novel antibody, which would facilitate early diagnosis and adequate treatment of the disease. 


\section{Cerebellar downbeat nystagmus: treatment with 4-aminopyridine}

Hypofunction of the cerebellar flocculus causes - among other ocular motor signs - downbeat nystagmus (DBN) that, in turn, may lead to debilitating vertical oscillopsia. In recent years, 4aminopyridine (4AP), a potassium channel blocker that increases Purkinje cell excitability, has become a valuable therapeutic option to dampen cerebellar DBN. 4AP also prevents attacks in episodic ataxia type 2 and improves cerebellar gait disorders. Fampridine is the sustained-release form of 4AP. Only two doses per day are required and cardiac side effects (prolonged QTc-time) are less common than in 4AP. Fampridine is usually well tolerated by patients [26].

In patients with ataxia-telangiectasia (A-T), which typically involves the cerebellum, Shaikh et al. studied the effect of 4AP on ocular motor signs [27]. 4AP reduced deficits ascribed to the loss of cerebellar Purkinje cells in these patients. Specifically, 4AP shortened the time constant of the horizontal vestibulo-ocular reflex and decreased spontaneous and periodic alternating nystagmus.

In a patient with a right posterior vermis lesion leading to positional DBN and vertigo, 4AP was beneficial [28]. The lesion had caused hypofunction of the nodulus and flocculus/paraflocculus, as demonstrated with FDG-PET. This hypofunction was partly reversed by $4 \mathrm{AP}$, which suggests that the cerebellar structures inhibiting the upward ocular drift bias were functionally restored by the substance.

Another patient with a cerebellar ocular motor syndrome showed a reversal of DBN into upbeat nystagmus (UBN) after a test dose of fampridine [29]. Most likely, fampridine caused a transient over-activity of Purkinje cell neurons in this patient. This, in turn, increased the inhibition of superior vestibular nuclei neurons, which predominantly encode in the upward direction. Consequently, the eyes drifted downward leading to UBN.

\section{Acknowledgement}

The authors are grateful to Klara Landau for her helpful comments.

\section{Highlights [box with bullet points]}

- Read-Right, a free web-based application (www.readright.ucl.ac.uk) improves reading in patients with right-sided hemianopia [4].

- The presence of nystagmus fast phases after caloric irrigation predicts consciousness recovery in patients with vegetative state on ICU [5].

- Anti-Kv1.4 [9] and anti-Lrp4 [11] are promising novel autoantibodies for the diagnosis of hitherto 'seronegative' myasthenia gravis.

- While cyclosporine A may be effective in the treatment of NMO [21], natalizumab may have deleterious effects [22].

- 4-aminopyridine (4AP) and fampridine (sustained-release form of 4AP) are effective treatments of spontaneous and positional downbeat nystagmus resulting from vestibulo-cerebellar hypofunction [26,27]. 


\section{Table 1}

Revised diagnostic criteria for chronic relapsing inflammatory optic neuropathy (CRION) [25]

1. History: optic neuritis and at least one relapse.

2. Clinical: Objective evidence for loss of visual function.

3. Labor: NMO-IgG seronegative.

4. Imaging: Contrast enhancement of the acutely inflamed optic nerves (optic nerve atrophy at later stages).

5. Treatment: Response to immunosuppressive treatment and relapse on withdrawal or dose reduction. 


\section{References}

1. Leigh RJ, Zee DS (2006) The neurology of eye movements, vol 4th. Oxford Press, New York

2. Tseng P-H, Cameron IM, Pari G, Reynolds J, Munoz D, Itti L (2013) High-throughput classification of clinical populations from natural viewing eye movements. Journal of neurology 260 (1):275-284. doi:10.1007/s00415-012-6631-2

3. Linder J, Wenngren B-I, Stenlund H, Forsgren L (2012) Impaired oculomotor function in a community-based patient population with newly diagnosed idiopathic parkinsonism. Journal of neurology 259 (6):1206-1214. doi:10.1007/s00415-011-6338-9

4. Ong Y-H, Brown M, Robinson P, Plant G, Husain M, Leff A (2012) Read-Right: a "web app" that improves reading speeds in patients with hemianopia. Journal of neurology 259 (12):2611-2615. doi:10.1007/s00415-012-6549-8

5. Weiss N, Tadie J-M, Faugeras F, Diehl J-L, Fagon J-Y, Guerot E (2012) Can fastcomponent of nystagmus on caloric vestibulo-ocular responses predict emergence from vegetative state in ICU? Journal of neurology 259 (1):70-76. doi:10.1007/s00415-011-6120-z 6. Trojano L, Moretta P, Loreto V, Cozzolino A, Santoro L, Estraneo A (2012) Quantitative assessment of visual behavior in disorders of consciousness. Journal of neurology 259 (9):1888-1895. doi:10.1007/s00415-012-6435-4

7. Finke C, Pech L, Sömmer C, Schlichting J, Stricker S, Endres M, Ostendorf F, Ploner C, Brandt A, Paul F (2012) Dynamics of saccade parameters in multiple sclerosis patients with fatigue. Journal of neurology 259 (12):2656-2663. doi:10.1007/s00415-012-6565-8

8. Suzuki S, Satoh T, Yasuoka H, Hamaguchi Y, Tanaka K, Kawakami Y, Suzuki N, Kuwana M (2005) Novel autoantibodies to a voltage-gated potassium channel Kv1.4 in a severe form of myasthenia gravis. Journal of neuroimmunology 170 (1-2):141-149.

doi:10.1016/j.jneuroim.2005.08.017

9. Romi F, Suzuki S, Suzuki N, Petzold A, Plant G, Gilhus N (2012) Anti-voltage-gated potassium channel Kv1.4 antibodies in myasthenia gravis. Journal of neurology 259 (7):13121316. doi:10.1007/s00415-011-6344-y

10. Higuchi O, Hamuro J, Motomura M, Yamanashi Y (2011) Autoantibodies to low-density lipoprotein receptor-related protein 4 in myasthenia gravis. Annals of neurology 69 (2):418422. doi:10.1002/ana.22312

11. Pevzner A, Schoser B, Peters K, Cosma N-C, Karakatsani A, Schalke B, Melms A, Kröger S (2012) Anti-LRP4 autoantibodies in AChR- and MuSK-antibody-negative myasthenia gravis. Journal of neurology 259 (3):427-435. doi:10.1007/s00415-011-6194-7 12. Rajakulendran S, Viegas S, Spillane J, Howard R (2012) Clinically biphasic myasthenia gravis with both AChR and MuSK antibodies. Journal of neurology 259 (12):2736-2739. doi:10.1007/s00415-012-6661-9

13. Devic E (1894) Myélite subaiguë compliquée de névrite optique. Bull Med (Paris) 8:1033-1034

14. Jarius S, Wildemann B (2012) The case of the Marquis de Causan (1804): an early account of visual loss associated with spinal cord inflammation. Journal of neurology 259 (7):1354-1357. doi:10.1007/s00415-011-6355-8

15. Jarius S, Wildemann B (2013) On the contribution of Thomas Clifford Allbutt, F.R.S., to the early history of neuromyelitis optica. Journal of neurology 260 (1):100-104. doi:10.1007/s00415-012-6594-3

16. Jacob A, Panicker J, Lythgoe D, Elsone L, Mutch K, Wilson M, Das K, Boggild M (2013) The epidemiology of neuromyelitis optica amongst adults in the Merseyside county of United Kingdom. Journal of neurology:1-4. doi:10.1007/s00415-013-6926-y 
17. Uzawa A, Mori M, Muto M, Masuda S, Kuwabara S (2012) When is neuromyelitis optica diagnosed after disease onset? Journal of neurology 259 (8):1600-1605. doi:10.1007/s00415011-6384-3

18. Wingerchuk DM, Lennon VA, Pittock SJ, Lucchinetti CF, Weinshenker BG (2006)

Revised diagnostic criteria for neuromyelitis optica. Neurology 66 (10):1485-1489.

doi:10.1212/01.wnl.0000216139.44259.74

19. Iorio R, Damato V, Mirabella M, Evoli A, Marti A, Plantone D, Frisullo G, Batocchi A (2013) Distinctive clinical and neuroimaging characteristics of longitudinally extensive transverse myelitis associated with aquaporin-4 autoantibodies. Journal of neurology:1-7. doi:10.1007/s00415-013-6997-9

20. Sellner J, Boggild M, Clanet M, Hintzen RQ, Illes Z, Montalban X, Du Pasquier RA, Polman CH, Sorensen PS, Hemmer B (2010) EFNS guidelines on diagnosis and management of neuromyelitis optica. European journal of neurology : the official journal of the European Federation of Neurological Societies 17 (8):1019-1032. doi:10.1111/j.1468-

1331.2010.03066.x

21. Kageyama T, Komori M, Miyamoto K, Ozaki A, Suenaga T, Takahashi R, Kusunoki S, Matsumoto S, Kondo T (2013) Combination of cyclosporine A with corticosteroids is effective for the treatment of neuromyelitis optica. Journal of neurology 260 (2):627-634. doi:10.1007/s00415-012-6692-2

22. Juryńczyk M, Zaleski K, Selmaj K (2013) Natalizumab and the development of extensive brain lesions in neuromyelitis optica. Journal of neurology 260 (7):1919-1921.

doi:10.1007/s00415-013-6965-4

23. Horwitz H, Degn M, Modvig S, Larsson HW, Wanscher B, Frederiksen J (2012) CSF abnormalities can be predicted by VEP and MRI pathology in the examination of optic neuritis. Journal of neurology 259 (12):2616-2620. doi:10.1007/s00415-012-6551-1 24. Kidd D, Burton B, Plant GT, Graham EM (2003) Chronic relapsing inflammatory optic neuropathy (CRION). Brain : a journal of neurology 126 (Pt 2):276-284

25. Petzold A, Plant G (2013) Chronic relapsing inflammatory optic neuropathy: a systematic review of 122 cases reported. Journal of neurology:1-10. doi:10.1007/s00415-013-6957-4 26. Claassen J, Feil K, Bardins S, Teufel J, Spiegel R, Kalla R, Schneider E, Jahn K, Schniepp R, Strupp M (2013) Dalfampridine in patients with downbeat nystagmus - an observational study. Journal of neurology:1-5. doi:10.1007/s00415-013-6911-5

27. Shaikh AG, Marti S, Tarnutzer AA, Palla A, Crawford TO, Zee DS, Straumann D (2013) Effects of 4-aminopyridine on nystagmus and vestibulo-ocular reflex in ataxia-telangiectasia. Journal of neurology. doi:10.1007/s00415-013-7046-4

28. Kremmyda O, Zwergal A, Fougère C, Brandt T, Jahn K, Strupp M (2013) 4-

Aminopyridine suppresses positional nystagmus caused by cerebellar vermis lesion. Journal of neurology 260 (1):321-323. doi:10.1007/s00415-012-6737-6

29. Feil K, Claaßen J, Bardins S, Teufel J, Habs M, Kalla R, Strupp M (2013) Transition from downbeat to upbeat nystagmus caused by 4-aminopyridine. Journal of neurology 260

(5):1426-1428. doi:10.1007/s00415-013-6907-1 